\title{
Central and Obstructive Apneas in Heart Failure With Reduced, Mid-Range and Preserved Ejection Fraction
}

\author{
Chiara Borrelli ${ }^{1,2,3}$, Francesco Gentile ${ }^{1}$, Paolo Sciarrone ${ }^{1}$, Gianluca Mirizzi ${ }^{1,2}$, \\ Giuseppe Vergaro ${ }^{1,2}$, Nicolò Ghionzoli ${ }^{1}$, Francesca Bramanti ${ }^{1}$, Giovanni ludice ${ }^{1}$, \\ Claudio Passino ${ }^{1,2}$, Michele Emdin ${ }^{1,2}$ and Alberto Giannoni ${ }^{1,2 *}$ \\ ${ }^{1}$ Fondazione Toscana G. Monasterio, Pisa, Italy, ${ }^{2}$ Institute of Life Sciences, Scuola Superiore Sant'Anna, Pisa, Italy, \\ ${ }^{3}$ Emergency Medicine Division, University of Pisa, Pisa, Italy
}

OPEN ACCESS

Edited by:

Filippo M. Sarullo,

Ospedale Buccheri la Ferla

Fatebenefratelli, Italy

Reviewed by:

Kenichi Hongo,

Jikei University School of

Medicine, Japan

Yoshitaka Iwanaga,

Kyoto University Hospital, Japan

*Correspondence:

Alberto Giannon

alberto.giannoni@gmail.com

Specialty section:

This article was submitted to Heart Failure and Transplantation,

a section of the journal

Frontiers in Cardiovascular Medicine

Received: 31 May 2019

Accepted: 12 August 2019 Published: 06 September 2019

Citation: Borrelli C, Gentile F, Sciarrone P, Mirizzi G, Vergaro G, Ghionzoli N, Bramanti F, ludice G, Passino C, Emdin M and Giannoni A (2019) Central and Obstructive Apneas in

Heart Failure With Reduced, Mid-Range and Preserved Ejection

Fraction.

Front. Cardiovasc. Med. 6:125 doi: 10.3389/fcvm.2019.00125
Background: Although central apneas (CA) and obstructive apneas $(\mathrm{OA})$ are highly prevalent in heart failure (HF), a comparison of apnea prevalence, predictors and clinical correlates in the whole HF spectrum, including HF with reduced ejection fraction (HFrEF), mid-range EF (HFmrEF) and preserved EF (HFpEF) has never been carried out so far.

Materials and methods: $700 \mathrm{HF}$ patients were prospectively enrolled and then divided according to left ventricular EF (408 HFrEF, $117 \mathrm{HFmrEF,} 175 \mathrm{HFpEF}$ ). All patients underwent a thorough evaluation including: 2D echocardiography; 24-h Holter-ECG monitoring; cardiopulmonary exercise testing; neuro-hormonal assessment and 24-h cardiorespiratory monitoring.

Results: In the whole population, prevalence of normal breathing (NB), CA and OA at daytime was 40, 51, and 9\%, respectively, while at nighttime 15, 55, and 30\%, respectively. When stratified according to left ventricular EF, CA prevalence decreased (daytime: 57 vs. 43 vs. $42 \%, p=0.001$; nighttime: 66 vs. 48 vs. $34 \%, p<0.0001$ ) from HFrEF to HFmrEF and HFpEF, while OA prevalence increased (daytime: 5 vs. 8 vs. $18 \%$, $p<0.0001$; nighttime 20 vs. 29 vs. $53 \%, p<0.0001$ ).

In HFrEF, male gender and body mass index (BMI) were independent predictors of both $\mathrm{CA}$ and $\mathrm{OA}$ at nighttime, while age, New York Heart Association functional class and diastolic dysfunction of daytime CA. In HFmrEF and HFpEF male gender and systolic pulmonary artery pressure were independent predictors of CA at daytime, while hypertension predicted nighttime OA in HFpEF patients; no predictor of nighttime CA was identified. When compared to patients with NB, those with CA had higher neuro-hormonal activation in all HF subgroups. Moreover, in the HFrEF subgroup, patients with CA were older, more comorbid and with greater hemodynamic impairment while, in the HFmrEF and HFpEF subgroups, they had higher left atrial volumes and more severe diastolic dysfunction, respectively. When compared to patients with NB, those with OA were older and more comorbid independently from background EF. 
Conclusions: Across the whole spectrum of HF, CA prevalence increases and OA decreases as left ventricular systolic dysfunction progresses. Different predictors and specific clinical characteristics might help to identify patients at risk of developing CA or $\mathrm{OA}$ in different HF phenotypes.

\section{Keywords: heart failure, heart failure with reduced ejection fraction, heart failure with mid-range ejection fraction,} heart failure with preserved ejection fraction, obstructive apneas, central apneas

\section{INTRODUCTION}

Apneas, either in the form of obstructive apneas $(\mathrm{OA})$ or central apneas (CA), are frequently observed in patients with heart failure and reduced ejection fraction (HFrEF, defined by a left ventricular ejection fraction-LVEF, <40\%) (1). In this setting, several studies have defined the prevalence of apneas (2-5), their clinical significance $(2,6-8)$ and impact on outcome $(2,4,6,7,9$, $10)$, especially when present also during the daytime $(2,5,6,10)$.

In HFrEF patients, CA are present both at nighttime-with a prevalence range of $20-70 \%(1-3)$ - and at daytime - with a prevalence range of $16-60 \%(5,6)$-depending on the apneahypopnea index (AHI) threshold used and the severity of the HF population recruited. Predictors of CA in HFrEF patients identified in different studies were age, male gender, body mass index (BMI), LVEF, atrial fibrillation, awake partial pressure of carbon dioxide and increased natriuretic peptides $(5,11,12)$. In HFrEF, CA have been associated with worse symptoms $(1,4,6)$, reduced exercise tolerance and peak oxygen consumption (3, $5,7)$, increased ventilatory inefficiency on effort, worse left ventricular (LV) systolic and diastolic function $(2,5)$, increased sympathetic activation and plasma levels of natriuretic peptides $(2,5)$ as well as increased incidence of atrial and ventricular arrhythmias $(13,14)$. Moreover, CA have been associated with an increased risk of mortality, both for HF progression and lifethreating arrhythmias $(2,4,6,7,9,10)$, especially when CA are present also during the daytime $(2,5,6,10)$.

On the other hand, in HFrEF patients, OA are present mainly at nighttime, with a prevalence range of $13-40 \%$ (2, 15). Predictors of OA in HFrEF are BMI and concomitant hypertension $(11,16)$, while, clinically, OA have been associated with higher LV and right ventricular function and exercise tolerance when compared to HFrEF patients with CA $(2,16)$. Finally, OA also carry prognostic significance in patients with HFrEF, even though mortality risk seems lower than that experienced by patients with CA $(2,17)$.

The epidemiological and clinical relevance of $\mathrm{OA}$ and $\mathrm{CA}$ in patients with $\mathrm{HF}$ and mid-range ejection fraction (HFmrEF, defined by LVEF between 40 and 49\%) (1) and HF and preserved

\footnotetext{
Abbreviations: AHI, apnea hypopnea index; BMI, body mass index; CA, central apneas; CAI, central apnea index HF, heart failure; HFmrEF, heart failure with mid-range ejection fraction; HFpEF, heart failure with preserved ejection fraction; HFrEF, heart failure with reduced ejection fraction; IR, interquartile range; LA, left atrium; LV, left ventricular; LVEF, left ventricular ejection fraction; MR, mitral regurgitation; NB, normal breathers; NT-proBNP, N-terminal fraction of pro-Btype natriuretic peptide; NYHA, New York Heart Association; OA, obstructive apneas; OAI, obstructive apnea index; $\mathrm{SaO}_{2}$, oxygen saturation; T90, time spent with an oxygen saturation below $90 \%$.
}

ejection fraction (HFpEF, defined by LVEF $\geq 50 \%$ ) (1) have been poorly addressed so far. In a study on 244 inpatients with HFpEF (defined with an LVEF > 55\%), OA were found in 39.8\% and were associated with higher rate of comorbidities, while CA were observed in $29.5 \%$ of patients and were associated with worse diastolic function, higher filling pressures, left atrial dimensions and natriuretic peptides levels (18). Results from another study, evaluating 115 outpatients with HF (38\% of whom $-n=43-$ had either HFmrEF or HFpEF defined with by a LVEF $\geq 45 \%$ ), reported a $62 \%$ prevalence of $\mathrm{OA}$ and $18 \%$ prevalence of CA (19), albeit no information on daytime apneas, $\mathrm{CA} / \mathrm{OA}$ predictors and clinical correlates was provided.

Therefore, given the lack of knowledge in this field and hypothesizing a potential clinical impact of both $\mathrm{OA}$ and $\mathrm{CA}$ also in patients with HFmrEF and HFpEF the aim of this study was to investigate the prevalence, predictors and clinical significance of $\mathrm{OA}$ and CA across the whole spectrum of HF.

\section{METHODS}

\section{Patient Population}

In the current study, we incorporated 525 patients with systolic HF (LVEF $<50 \%$ ) of a previously published study (2). Those patients were then divided according to 2016 ESC HF guidelines (1) based on the underlying LVEF (408 patients were classified as HFrEF and 117 patients were classified as HFmrEF).

In the same period (from January 2006 to December 2013), a population of 175 consecutive patients with HFpEF was also enrolled. For both patients with HFmrEF and HFpEF the presence of additional guidelines-recommended criteria (signs/symptoms of HF, elevated plasmatic concentrations of natriuretic peptides or relevant structural heart disease or diastolic dysfunction) was also considered (1).

Exclusion criteria were: acute coronary syndromes or episodes of acute HF within 3 months; severe pulmonary, renal or neurological disease; therapy with drugs affecting the respiratory drive such as morphine or derivatives, theophylline, oxygen, benzodiazepines, acetazolamide, or treatment with continuous positive airway pressure or servoventilation. Written informed consent was obtained from each patient before enrolment, and the study was approved by the Institutional Ethics Committee and conducted in accordance with Declaration of Helsinki of the World Medical Association.

\section{Study Design}

All patients underwent a comprehensive evaluation $(2,20)$ with: 2-dimensional transthoracic echocardiography (IE33 
ultrasound machine, Philips Medical Systems, Palo Alto, California), 24-h ECG Holter recording (Elamedical, Paris, France), symptom-limited cardiopulmonary exercise testing (VMAX, Sensormedics, Conshohocken, Pennsylvania, US) and biohumoral characterization, including assessment of plasma levels of norepinephrine, aldosterone, renin activity and N-terminal fraction of pro-B-type natriuretic peptide (NT-proBNP). Finally, all patients also underwent 24-h cardiorespiratory polygraphic recording for screening of $\mathrm{CA} / \mathrm{OA}$, as previously reported (2) and as explained below. All examinations were performed within 3-days in condition of clinical stability and without altering HF related treatments.

\section{4-h Cardiorespiratory Polygraphic Recording}

All patients underwent a 24-h continuous polygraphic recording to identify respiratory events and to classify them as central or obstructive. According to the latest guidelines of the American Academy of Sleep Medicine (21), three essential signals were incorporated: (1) nasal airflow; (2) chest and abdominal respiratory movements by inductance plethysmography; (3) oxygen saturation $\left(\mathrm{SaO}_{2}\right)$ (Somté PSG2; Compumedics).

An apnea was defined as a cessation of breathing lasting $>10 \mathrm{~s}$; presence or absence of thoracic and abdominal movements allowed distinction between central and obstructive events, respectively. A hypopnea was defined as a reduction in airflow $>50 \%$ of normal, lasting $>10$ s, with a $\mathrm{SaO}_{2}$ reduction $\geq 4 \%$ (21).

The presence/absence of a respiratory disturbance was established by means of the AHI (22). CA were diagnosed in presence of an AHI $\geq 5$ events/h, with $>50 \%$ of apneic events being central, whereas a diagnosis of OA was made in presence of an AHI $\geq 5$ events/h, with $>50 \%$ of apneic events being obstructive (22). CA/OA severity was then classified as being: mild with an AHI $\geq 5$ and $<15$ events/h, moderatesevere with an AHI $\geq 15$ and $<30$ events/h, and severe with an $\mathrm{AHI} \geq 30$ events/h. Given the potential misclassification of hypopneas without the use of esophageal pressure transducer or diaphragmatic electromyography and the poor reliability of indirect algorithms (23), we considered hypopneas to follow the distribution of the majority of the apneic events (24). Nonetheless, we also performed an analysis exclusively based on the apneic events using the central apnea index (number of CA per hour, CAI) and the obstructive apnea index (number of OA per hour, OAI). Finally, we also evaluated the burden of desaturation with the minimum $\mathrm{SaO}_{2}$ and the time spent with a $\mathrm{SaO}_{2}<90 \%$ (T90).

The AHI, CAI, and OAI were computed over the whole 24$\mathrm{h}$ recording, at night (10 p.m. to 6:59 a.m.) and during daytime (7 a.m. to 9:59 p.m.), as previously reported (2). Data analysis was performed by experienced sleep technicians (G.I. and F.B.), and then controlled by a physician with specific relevant clinical and research experience in the field (A.G., C.B., M.E., or C.P.).

\section{Statistical Analysis}

Statistical analysis was performed using SPSS 21.0 program (1989-2012, LEAD technologies Inc., USA). Values were presented as mean \pm standard deviation (SD) for variables with normal distribution, median and interquartile range (IR) for variables with skewed distribution and as percentage for categorical data. Variables with a skewed distribution were first log transformed before regression analysis. To address predictors of OA/CA, univariate logistic regression analysis was first performed. Statistically significant variables $(p<0.05)$ were than manually inserted in a multivariable logistic regression analysis to identify independent predictors of OA/CA. For continuous variables, differences between 2 groups were evaluated through the independent student $T$-test and Mann-Whitney $U$ test, while differences among $>2$ groups were evaluated through ANOVA or Kruskal-Wallis one-way analysis of variance, with Bonferroni post-hoc correction. For categorical variables, differences were analyzed with the Chi-Square with Yates correction or Fisher's exact test, as appropriate. A two-tailed $p$-value $<0.05$ was considered statistically significant.

\section{RESULTS}

\section{Patient Population}

The clinical characteristics of the general population and the different HF subgroups (700 patients, 408 with HFrEF, 117 with HFmrEF and 175 with HFpEF) are summarized in Table 1.

Dilated cardiomyopathy was the most prevalent HF etiology in HFrEF and HFmrEF ( $p<0.001$ vs. HFpEF), while cardiomyopathy of other etiology (i.e., valvular disorders, amyloidosis, hypertrophic cardiomyopathy) was more prevalent in HFpEF $(p<0.001)$. Patients with HFpEF were older $(p<$ 0.0001 ) and had more comorbidities than patients with HFrEF (namely: atrial fibrillation, hypertension, chronic obstructive pulmonary disease, anemia; all $p<0.05$ ).

Patients with HFrEF had worse hemodynamic compromise compared to the other HF subgroups, as expressed by more severe diastolic dysfunction and increased pulmonary artery pressure (vs. both HFmrEF and HFpEF, all $p<0.05$ ), greater left atrium (LA) volume and prevalence of moderate-severe mitral regurgitation (MR) (vs. HFmrEF, all $p<0.05$ ), and worse right ventricular function (vs. HFpEF, $p<0.001$ ).

Furthermore, patients with HFrEF had higher values of NT-proBNP and plasma renin activity when compared to both HFmrEF and HFpEF (both $p<0.001$ ), and higher norepinephrine when compared to HFmrEF only $(p=0.02)$. Patients with HFrEF were more frequently treated with mineralocorticoid receptor antagonists and ICD/CRT-D than patients with HFmrEF and HFpEF (both $p<0.001$ ), while patients with $\mathrm{HFpEF}$ were more often treated with loop diuretics than patients with HFrEF and HFmrEF (all $p<0.05$ ).

\section{Distribution of Apneas During the 24-h Across the Whole HF Spectrum}

Prevalence rates of patients with NB, OA and CA during daytime, nighttime and 24-h in the whole population and in the three HF phenotypes are shown in Figure 1, while data on the AHI, CAI, OAI, minimal $\mathrm{SaO}_{2}$ and $\mathrm{T} 90$ are reported in Table 2.

In the whole population, using an AHI cut-off $\geq 5$ events/h, the prevalence of $\mathrm{NB}, \mathrm{CA}$, and $\mathrm{OA}$ was 40,51 , and $9 \%$ at daytime and 15,55 , and $30 \%$ at nighttime, respectively. 
TABLE 1 | Characteristics of general population.

\begin{tabular}{|c|c|c|c|c|}
\hline & $\begin{array}{l}\text { All patients } \\
\qquad N=700\end{array}$ & $\begin{array}{c}\text { HFrEF } \\
N=408\end{array}$ & $\begin{array}{l}\text { HFmrEF } \\
N=117\end{array}$ & $\begin{array}{l}\text { HFpEF } \\
N=175\end{array}$ \\
\hline Age (years) & $66.8 \pm 12.4$ & $65.3 \pm 12.9$ & $67.3 \pm 11.9$ & $71.4 \pm 9.8^{d}$ \\
\hline $\mathrm{BMI}\left(\mathrm{kg} / \mathrm{m}^{2}\right)$ & $28.3 \pm 5.4$ & $27.2 \pm 5.1$ & $28.6 \pm 5.2$ & $31.4 \pm 8.7^{\mathrm{d}, \mathrm{f}}$ \\
\hline NYHA I-II/III-IV (\%) & $67 / 33$ & $63 / 37$ & $76 / 24$ & $70 / 30$ \\
\hline Other etiology (\%) & 19 & 2 & 3 & $70^{d, f}$ \\
\hline \multicolumn{5}{|l|}{ Comorbidities } \\
\hline Atrial fibrillation (\%) & 29 & 24 & 31 & $40^{c}$ \\
\hline Systemic hypertension (\%) & 59 & 50 & 63 & $75^{d}$ \\
\hline Diabetes mellitus (\%) & 31 & 30 & 28 & 35 \\
\hline Creatinine (mg/dL) & $1.2 \pm 0.5$ & $1.2 \pm 0.5$ & $1.2 \pm 0.6$ & $1.1 \pm 0.5^{\mathrm{c}}$ \\
\hline $\operatorname{eGFR}\left(\mathrm{mL} / \mathrm{min} / 1.73 \mathrm{~m}^{2}\right)$ & $64(49-81)$ & $62(48-78)$ & $67(49-87)$ & $67(51-83)$ \\
\hline $\mathrm{TSH}(\mu \mathrm{Ul} / \mathrm{mL})$ & $1.8(1.1-2.8)$ & $1.8(1.1-2.8)$ & $1.5(0.9-2.6)$ & $2.0(1.3-3.0)$ \\
\hline C-reactive protein (mg/dL) & $0.3(0.1-0.8)$ & $0.3(0.1-0.8)$ & $0.3(0.1-0.8)$ & $0.3(0.2-1.0)$ \\
\hline \multicolumn{5}{|l|}{ Echocardiography } \\
\hline LVEF (\%) & $37.9 \pm 13.9$ & $28.3 \pm 6.8$ & $43.1 \pm 2.8^{b}$ & $57.3 \pm 6.8^{\mathrm{d}, \mathrm{f}}$ \\
\hline LA volume $\left(\mathrm{mL} / \mathrm{m}^{2}\right)$ & $40.3 \pm 14.2$ & $40.6 \pm 12.8$ & $36.6 \pm 11.3^{a}$ & $39.1 \pm 11.0$ \\
\hline Diastolic dysfunction II-III (\%) & 29 & 48 & $30^{\mathrm{a}}$ & $21^{d}$ \\
\hline Moderate-severe MR (\%) & 43 & 48 & $28^{\mathrm{a}}$ & 40 \\
\hline TAPSE (mm) & $18.5 \pm 5.0$ & $17.7 \pm 4.9$ & $18.7 \pm 5.1$ & $20.0 \pm 4.9^{d}$ \\
\hline Peak $\mathrm{VO}_{2}(\%)$ & $57.7 \pm 17.1$ & $54.7 \pm 16.4$ & $66.1 \pm 17.2^{b}$ & $60.8 \pm 16.6^{c}$ \\
\hline VENCO 2 slope & $33.2 \pm 8.1$ & $33.8 \pm 8.4$ & $30.2 \pm 6.7^{\mathrm{a}}$ & $33.9 \pm 5.5^{\mathrm{e}}$ \\
\hline \multicolumn{5}{|l|}{ Therapy } \\
\hline$\beta$-blockers (\%) & 89 & 96 & 96 & $68^{d, f}$ \\
\hline ACEi/ARB (\%) & 87 & 94 & 88 & $69^{\mathrm{d}, \mathrm{e}}$ \\
\hline MRA (\%) & 68 & 83 & $55^{\mathrm{b}}$ & $41^{d}$ \\
\hline Furosemide (\%) & 47 & 48 & 40 & $62^{\mathrm{c}, \mathrm{e}}$ \\
\hline ICD/CRT-D (\%) & $22 / 19$ & $35 / 26$ & $6 / 4^{b}$ & $2 / 13^{d}$ \\
\hline
\end{tabular}

ACEi, angiotensin-converting enzyme inhibitors; ARB, angiotensin II receptor blockers; BMI, body mass index; COPD, chronic obstructive pulmonary disease; CRP, C-reactive protein; CRT-D, cardiac resynchronization therapy with defibrillator; DCM, dilated cardiomyopathy; eGFR, estimated glomerular filtration rate (with the Modification of Diet in Renal Disease-MDRD formula); Hb, hemoglobin; Hs, high sensitive; ICD, implantable cardioverter defibrillator; ICM, ischemic cardiomyopathy; LA, left atrium; LVEF, left ventricular ejection fraction; LVMI, left ventricular mass index; MR, mitral regurgitation; MRA, mineralocorticoid receptor antagonists; NT-proBNP, N-terminal fraction of pro-B-type natriuretic peptide; NYHA, New York Heart Association; SPAP, systolic pulmonary artery pressure; PRA, plasmatic renin activity; TAPSE, tricuspid annular plane systolic excursion; TSH, thyroid stimulating hormone; VENVCO ${ }_{2}$, ventilation to carbon dioxide production ratio; $\mathrm{VO}_{2}$, oxygen consumption.

Values are expressed as mean \pm standard deviation for variables with normal distribution, median (interquartile range) for variables with skewed distribution and percentages for categorical variables.

${ }^{a} p<0.05$ HFrEF vs. HFMrEF.

${ }^{b} p<0.001$ HFrEF vs. HFMrEF.

${ }^{c} p<0.05$ HFrEF vs. HFpEF.

${ }^{d} p<0.001$ HFrEF vs. HFpEF.

${ }^{e} p<0.05$ HFMrEF vs. HFpEF.

${ }^{f} p<0.001$ HFMrEF vs. HFpEF. 

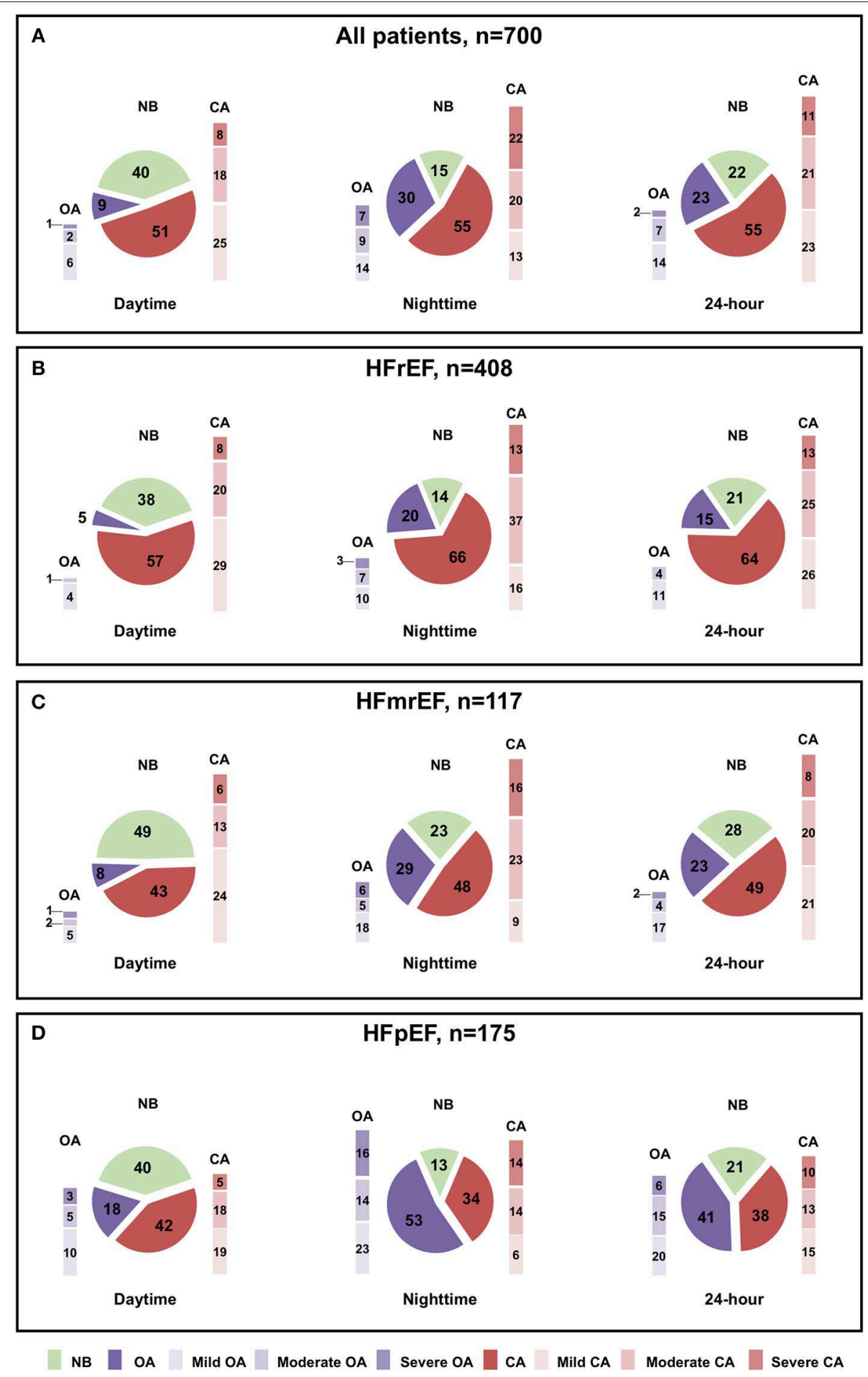

FIGURE 1 | Prevalence of normal breathing (NB), central apneas (CA) and obstructive apneas (OA) during daytime, nighttime and 24-h, across the whole heart failure (HF) spectrum. (A) Prevalence of NB, CA and OA in the general population of HF patients during daytime, nighttime and 24-h. (B) Prevalence of NB, CA and OA in HF with reduced ejection fraction (HFrEF) during daytime, nighttime and 24-h. (C) Prevalence of NB, CA, and OA in $\mathrm{HF}$ with mid-range ejection fraction (HFmrEF) during daytime, nighttime and 24-h. (D) Prevalence of NB, CA and OA in HF with preserved ejection fraction (HFpEF) during daytime, nighttime and 24-h.

When stratified according to LVEF, CA were more prevalent in HFrEF than HFmrEF and HFpEF both at daytime (57 vs. 43 vs. $42 \%$; comparison between groups, $p=0.001$ ) and nighttime (66 vs. 48 vs. $34 \%$; comparison between groups $p<0.0001$ ), while prevalence of $\mathrm{OA}$ was higher in HFpEF than in HFrEF and HFmrEF, both at daytime 
TABLE 2 | 24-h cardiorespiratory monitoring across the whole HF spectrum.

\begin{tabular}{|c|c|c|c|c|}
\hline & $\begin{array}{l}\text { All patients } \\
\quad N=700\end{array}$ & $\begin{array}{c}\text { HFrEF } \\
N=408\end{array}$ & $\begin{array}{l}\text { HFmrEF } \\
N=117\end{array}$ & $\begin{array}{c}\text { HFpEF } \\
N=175\end{array}$ \\
\hline Diurnal apnea prevalence (\%) & 60 & 62 & 51 & 60 \\
\hline 24-h apnea prevalence (\%) & 78 & 79 & 72 & 79 \\
\hline Diurnal CA prevalence (\%) & 51 & 57 & $43^{a}$ & $42^{b}$ \\
\hline Diurnal OA prevalence (\%) & 9 & 5 & 8 & $18^{b, c}$ \\
\hline Nocturnal OA prevalence (\%) & 30 & 20 & 29 & $53^{b, c}$ \\
\hline 24-h OA prevalence (\%) & 23 & 15 & 23 & $41^{b, c}$ \\
\hline Diurnal AHI (events/h) & $8(2-17)$ & $9(2-17)$ & $6(2-14)$ & $8(3-19)$ \\
\hline Nocturnal AHI (events/h) & $19(8-33)$ & $20(8-33)$ & $16(6-29)$ & $21(11-35)$ \\
\hline 24-h CAl (events/h) & $2(0-8)$ & $2(0-11)$ & $1(0-6)^{a}$ & $1(0-4)^{b}$ \\
\hline Diurnal OAI (events/h) & $0(0-1)$ & $0(0-0)$ & $0(0-1)$ & $0(0-1)^{b, c}$ \\
\hline Nocturnal OAI (events/h) & $0(0-2)$ & $0(0-1)$ & $0(0-3)^{a}$ & $1(0-5)^{b, c}$ \\
\hline 24-h OAl (events/h) & $0(0-2)$ & $0(0-0)$ & $0(0-2)^{a}$ & $1(0-3)^{b, c}$ \\
\hline $\mathrm{SaO}_{2} \min (\%)$ & $85(80-88)$ & $86(81-89)$ & $85(80-89)$ & $82(78-86)$ \\
\hline T90 (min) & $8(3-16)$ & $6(2-13)$ & $8(2-17)$ & $12(5-18)$ \\
\hline
\end{tabular}

AHI, apnea-hypopnea index; CA, central apneas; CAl, central apnea index; OA, obstructive apneas; OAl, obstructive apnea index; T90, time spent with an oxygen saturation below 90\%. Values are expressed as median (interquartile range) for variables with skewed distribution and percentages for categorical variables. ${ }^{a} p<0.05$ HFrEF vs. HFMrEF.

${ }^{b} p<0.001$ HFrEF vs. HFpEF.

${ }^{c} p<0.05$ HFMrEF vs. HFpEF.

(5 vs. 8 vs. $18 \%$; comparison between groups $p<0.0001$ ) and nighttime (20 vs. 29 vs. 53\%; comparison between groups, $p<0.0001)$.

Using an AHI cut-off for moderate-severe apneas $\geq 15$ events/h, daytime CA prevalence was similar between the three groups ( 28 vs. 19 vs. $23 \%$; $p$ between groups $=0.09$ ), while nighttime CA was still higher in HFrEF (50 vs. 39 vs. $28 \%$; $p$ between groups $<0.0001)$. Conversely, moderate-severe OA were less prevalent in HFrEF and HFmrEF than in HFpEF both at daytime ( 1 vs. 3 vs. $8 \%$; $p$ between groups $<0.001$ ) and nighttime (10 vs. 11 vs. $30 \%$; $p$ between groups $<0.0001$ ).

\section{Predictors of Apneas Across the Whole Spectrum of HF Predictors of Central Apneas}

Univariarable and multivariable predictors of CA at nighttime are shown in Table 3. In the whole population only male gender and LA volume remained independent predictors of nighttime CA (male gender: OR 10.95 CI 2.87-41.78, $p<0.001$; LA volume OR 1.09 CI 1.01-1.19, $p=0.04$ ). When stratified according to LVEF, in the HFrEF population male gender and BMI remained as independent predictors of CA at nighttime (male gender: OR 2.99 CI 1.18-7.63, $p=0.02$; BMI: OR 1.14 CI 1.02-1.26, $p=0.02$ ), while no predictors were found in HFmrEF and HFpEF populations.

Univariable and multivariable predictors of CA at daytime are shown in Table 4. In the whole population, age (OR 1.04 CI 1.01-1.08, $p=0.02$ ), male gender (OR 5.19 CI 2.42-11.14, $p<$ 0.0001 ) and LA volume (OR 1.04 CI 1.01-1.08, $p=0.03$ ) were found to be independent predictors of daytime CA. In the HFrEF population age (OR 1.09 CI 1.03-1.16, p = 0.04), functional NYHA class (OR 2.28 CI 1.08-4.38, $p=0.03$ ) and grade II-III diastolic dysfunction (OR 4.26 CI 1.16-15.69, $p=0.03$ ) remained as independent predictors, while sPAP (OR 1.07 CI 0.99-1.15, $p=0.04)$ was the only independent predictor in HFmrEF. Conversely, no predictor was identified in the HFpEF population.

\section{Predictors of Obstructive Apneas}

Considering the low prevalence of OA at daytime, the analysis of OA predictors was limited at the nighttime period.

Univariable and multivariable predictors of OA at nighttime are shown in Table 5. In the whole population, at multivariable logistic regression analysis, age (OR 1.04 CI 1.01-1.08, $p=0.03$ ), male gender (OR 7.11 CI 3.07-16.50, $p<0.001$ ) and BMI (OR 1.16 CI 1.06-1.27, $p=0.001$ ) resulted independent predictors of $\mathrm{OA}$ at nighttime. 
TABLE 3 | Predictors of central apneas at nighttime.

\begin{tabular}{|c|c|c|c|c|c|c|}
\hline & \multicolumn{3}{|c|}{ Univariate analysis } & \multicolumn{3}{|c|}{ Multivariate analysis } \\
\hline & OR & Cl & $P$-value & OR & Cl & $P$-value \\
\hline \multicolumn{7}{|l|}{ In the whole population } \\
\hline Age & 1.04 & $1.03-1.06$ & $<0.001$ & - & - & - \\
\hline Male gender & 4.09 & $2.58-6.49$ & $<0.001$ & 10.95 & $2.87-41.78$ & $<0.001$ \\
\hline NYHA class & 1.51 & $1.13-2.04$ & 0.006 & - & - & - \\
\hline ICM & 2.17 & $1.34-3.50$ & 0.001 & - & - & - \\
\hline Systemic hypertension & 1.80 & $1.16-2.79$ & 0.009 & - & - & - \\
\hline $\mathrm{Hb}$ & 1.16 & $1.02-1.32$ & 0.020 & - & - & - \\
\hline C-reactive protein & 1.23 & $1.03-1.46$ & 0.020 & - & - & - \\
\hline LVEF & 0.97 & $0.95-0.99$ & $<0.001$ & - & - & - \\
\hline LVMI & 1.01 & $1.00-1.01$ & 0.010 & - & - & - \\
\hline LA volume & 1.07 & $1.04-1.10$ & $<0.001$ & 1.09 & $1.01-1.19$ & 0.040 \\
\hline Diastolic dysfunction II-III & 3.23 & $1.82-5.73$ & $<0.001$ & - & - & - \\
\hline Severe MR & 2.56 & $1.13-5.79$ & 0.020 & - & - & - \\
\hline TAPSE & 0.93 & $0.89-0.98$ & 0.005 & - & - & - \\
\hline sPAP & 1.05 & $1.02-1.08$ & 0.001 & - & - & - \\
\hline NT-proBNP & 1.52 & $1.29-1.80$ & $<0.001$ & - & - & - \\
\hline Norepinephrine & 2.31 & $1.60-3.33$ & $<0.001$ & - & - & - \\
\hline ICD & 1.85 & $1.06-3.22$ & 0.03 & - & - & - \\
\hline \multicolumn{7}{|l|}{ In the HFrEF population } \\
\hline Age & 1.05 & $1.03-1.07$ & $<0.001$ & - & - & - \\
\hline Male gender & 4.32 & $2.36-7.88$ & $<0.001$ & 2.99 & $1.18-7.63$ & 0.020 \\
\hline BMl & 1.10 & $1.02-1.18$ & 0.009 & 1.14 & $1.02-1.26$ & 0.020 \\
\hline NYHA class & 1.60 & $1.09-2.35$ & 0.020 & - & - & - \\
\hline ICM & 3.27 & $1.68-6.32$ & $<0.001$ & - & - & - \\
\hline Atrial fibrillation & 2.45 & $1.12-5.46$ & 0.030 & - & - & - \\
\hline Systemic hypertension & 2.29 & $1.26-4.17$ & 0.007 & - & - & - \\
\hline Creatinine & 2.65 & $1.20-5.81$ & 0.020 & - & - & - \\
\hline eGFR & 0.99 & $0.98-1.00$ & 0.020 & - & - & - \\
\hline LVEF & 0.94 & $0.90-0.98$ & 0.008 & - & - & - \\
\hline LVMI & 1.01 & $1.00-1.02$ & 0.010 & - & - & - \\
\hline LA volume & 1.07 & $1.03-1.12$ & $<0.001$ & - & - & - \\
\hline Diastolic dysfunction II-III & 3.30 & $1.60-6.83$ & 0.001 & - & - & - \\
\hline \multicolumn{7}{|c|}{ In the HFmrEF population } \\
\hline Male gender & 6.60 & $2.01-21.44$ & 0.002 & - & - & - \\
\hline LA volume & 1.10 & $1.01-1.20$ & 0.020 & - & - & - \\
\hline sPAP & 1.12 & $1.01-1.23$ & 0.030 & - & - & - \\
\hline \multicolumn{7}{|l|}{ In the HFpEF population } \\
\hline Age & 1.07 & $1.00-1.14$ & 0.040 & - & - & - \\
\hline NT-proBNP & 1.70 & $1.12-2.58$ & 0.010 & - & - & - \\
\hline Norepinephrine & 4.84 & $1.31-17.83$ & 0.020 & - & - & - \\
\hline
\end{tabular}

BMI, body mass index; Cl, confidence interval; eGFR, estimated glomerular filtration rate (with the Modification of Diet in Renal Disease-MDRD formula); Hb, hemoglobin; ICD, implantable cardioverter defibrillator; ICM, ischemic cardiomyopathy; LA, left atrium; LVEF, left ventricular ejection fraction; LVMI, left ventricular mass index; MR, mitral regurgitation; NT-proBNP, N-terminal fraction of pro-B-type natriuretic peptide; NYHA, New York Heart Association; OR, odds ratio; SPAP, systolic pulmonary artery pressure; TAPSE, tricuspid annular plane systolic excursion.

Similarly, in the HFrEF population, at multivariable analysis, male gender (OR 3.53 CI 1.18-10.54, $p=0.03$ ) and BMI (OR 1.16 CI $1.03-1.30, p=0.02)$ remained the only independent predictors of $\mathrm{OA}$ at nighttime, while systemic hypertension in HFpEF (OR 2.84 CI 1.07-7.57, $p=0.04$ ). On the contrary, no independent predictor of nighttime OA was found in the HFmrEF population.

\section{Clinical Correlates of Apneas Across the Whole Spectrum of HF}

Clinical correlates of CA are summarized in Table 6 and Supplementary Tables 1-3.

Compared to NB, patients with HFrEF and both nighttime (Supplementary Table 1) and daytime (Supplementary Table 2) CA had more frequently atrial fibrillation, higher LV filling 
TABLE 4 | Predictors of central apneas at daytime.

\begin{tabular}{|c|c|c|c|c|c|c|}
\hline & \multicolumn{3}{|c|}{ Univariate analysis } & \multicolumn{3}{|c|}{ Multivariate analysis } \\
\hline & OR & $\mathrm{Cl}$ & $P$-value & OR & Cl & $P$-value \\
\hline \multicolumn{7}{|l|}{ In the whole population } \\
\hline Age & 1.03 & $1.02-1.05$ & $<0.0001$ & 1.04 & $1.01-1.08$ & 0.020 \\
\hline Male gender & 2.59 & $1.81-3.69$ & $<0.0001$ & 5.19 & $2.42-11.14$ & $<0.0001$ \\
\hline NYHA class & 1.51 & $1.23-1.85$ & $<0.0001$ & - & - & - \\
\hline ICM & 1.72 & $1.23-2.41$ & 0.002 & - & - & - \\
\hline Systemic hypertension & 1.41 & $1.02-1.95$ & 0.039 & - & - & - \\
\hline Diabetes mellitus & 1.39 & $1.01-1.93$ & 0.048 & - & - & - \\
\hline Creatinine & 1.70 & $1.18-2.46$ & 0.004 & - & - & - \\
\hline eGFR & 0.99 & $0.98-0.99$ & 0.001 & - & - & - \\
\hline LVEF & 0.98 & $0.97-0.99$ & $<0.0001$ & - & - & - \\
\hline LVMI & 1.01 & $1.00-1.01$ & 0.004 & - & - & - \\
\hline LA volume & 1.04 & $1.02-1.06$ & $<0.0001$ & 1.04 & $1.01-1.08$ & 0.030 \\
\hline Diastolic dysfunction II-III & 3.40 & $2.25-5.14$ & $<0.0001$ & - & - & - \\
\hline Moderate-severe MR & 1.71 & $1.01-2.89$ & 0.044 & - & - & - \\
\hline SPAP & 1.02 & $1.00-1.04$ & 0.033 & - & - & - \\
\hline \multicolumn{7}{|l|}{ In the HFrEF population } \\
\hline Age & 1.05 & $1.04-1.07$ & $<0.0001$ & 1.09 & $1.03-1.16$ & 0.040 \\
\hline Male gender & 2.34 & $1.45-3.79$ & 0.001 & - & - & - \\
\hline BMI & 1.05 & $1.00-1.10$ & 0.031 & - & - & - \\
\hline NYHA class & 1.77 & $1.33-2.36$ & $<0.0001$ & 2.28 & $1.08-4.38$ & 0.030 \\
\hline ICM & 2.18 & $1.42-3.36$ & $<0.0001$ & - & - & - \\
\hline Atrial fibrillation & 1.88 & $1.14-3.13$ & 0.014 & - & - & - \\
\hline Diabetes mellitus & 1.59 & $0.99-2.54$ & 0.050 & - & - & - \\
\hline Creatinine & 2.59 & $1.51-4.45$ & 0.001 & - & - & - \\
\hline eGFR & 0.98 & $0.97-0.99$ & $<0.001$ & - & - & - \\
\hline C-reactive protein & 1.21 & $1.02-1.43$ & 0.025 & - & - & - \\
\hline LVEF & 0.96 & $0.93-0.99$ & 0.014 & - & - & - \\
\hline LA volume & 1.06 & $1.03-1.09$ & $<0.001$ & - & - & - \\
\hline Diastolic dysfunction II-III & 4.39 & $2.60-7.41$ & $<0.001$ & 4.26 & $1.16-15.69$ & 0.030 \\
\hline SPAP & 1.03 & $1.01-1.05$ & 0.012 & - & - & - \\
\hline$\beta$-blockers & 3.17 & $1.04-9.66$ & 0.040 & - & - & - \\
\hline \multicolumn{7}{|c|}{ In the HFmrEF population } \\
\hline Male gender & 2.65 & $0.99-7.07$ & 0.040 & - & - & - \\
\hline LVMI & 1.02 & $1.00-1.04$ & 0.015 & - & - & - \\
\hline SPAP & 1.06 & $1.00-1.13$ & 0.044 & 1.07 & $0.99-1.15$ & 0.040 \\
\hline \multicolumn{7}{|l|}{ In the HFpEF population } \\
\hline Male gender & 3.00 & $1.51-6.09$ & 0.002 & - & - & - \\
\hline
\end{tabular}

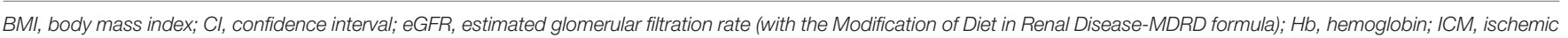

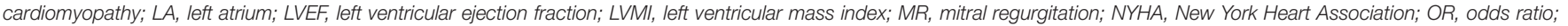
SPAP, systolic pulmonary artery pressure; TAPSE, tricuspid annular plane systolic excursion.

pressures (as expressed by worse diastolic dysfunction and greater LA volume (all $p<0.001$ ) and greater values of NT-proBNP and plasma norepinephrine (all $p<0.001$ ), despite similar neuro-hormonal antagonism therapy. Patients with HFmrEF, on the other hand, had greater LA volume when compared to NB $(p<0.05)$, while patients with HFpEF had higher LV filling pressures and increased plasma levels of norepinephrine and NT-proBNP (all $p<0.05$ ) (Supplementary Tables 1, 2).
When comparing patients with CA limited to the nighttime period with those showing CA both during the daytime and nighttime (using a cutpoint of $\mathrm{AHI} \geq 15$ events/h) (Table 6), the latter showed worse symptoms (higher NYHA class), greater diastolic dysfunction and higher NT-proBNP in HFrEF patients (all $p<0.05$ ). Patients with CA during the day and the night also showed higher norepinephrine plasma levels and worse oxygen saturation profile, as expressed by a higher time spent with a $\mathrm{SaO}_{2}$ below 90\% (T90), both in patients with HFrEF and HFpEF (all 
TABLE 5 | Predictors of obstructive apneas at nighttime.

\begin{tabular}{|c|c|c|c|c|c|c|}
\hline & \multicolumn{3}{|c|}{ Univariate analysis } & \multicolumn{3}{|c|}{ Univariate analysis } \\
\hline & OR & Cl & $P$-value & OR & Cl & $P$-value \\
\hline \multicolumn{7}{|c|}{ In the whole population } \\
\hline Age & 1.05 & $1.03-1.07$ & $<0.001$ & 1.04 & $1.01-1.08$ & 0.030 \\
\hline Male gender & 2.64 & $1.61-4.33$ & $<0.001$ & 7.11 & $3.07-16.50$ & $<0.001$ \\
\hline BMI & 1.15 & $1.09-1.21$ & $<0.001$ & 1.16 & $1.06-1.27$ & 0.001 \\
\hline NYHA class & 1.45 & $1.04-2.00$ & 0.030 & - & - & - \\
\hline Systemic hypertension & 2.85 & $1.75-4.65$ & $<0.001$ & - & - & - \\
\hline COPD & 2.00 & $1.03-3.93$ & 0.040 & - & - & - \\
\hline LVEF & 1.03 & $1.01-1.05$ & 0.002 & - & - & - \\
\hline LA volume & 1.03 & $1.01-1.07$ & 0.020 & - & - & - \\
\hline \multicolumn{7}{|c|}{ In the HFrEF population } \\
\hline Age & 1.04 & $1.01-1.06$ & 0.004 & - & - & - \\
\hline Male gender & 3.73 & $1.76-7.92$ & 0.001 & 3.53 & $1.18-10.54$ & 0.030 \\
\hline $\mathrm{BMl}$ & 1.13 & $1.05-1.22$ & 0.001 & 1.16 & $1.03-1.30$ & 0.020 \\
\hline NYHA class & 1.60 & $1.02-2.51$ & 0.040 & - & - & - \\
\hline ICM & 2.98 & $1.40-6.35$ & 0.005 & - & - & - \\
\hline Systemic hypertension & 2.52 & $1.24-5.11$ & 0.010 & - & - & - \\
\hline LA volume & 1.05 & $1.01-1.09$ & 0.030 & - & - & - \\
\hline \multicolumn{7}{|c|}{ In the HFmrEF population } \\
\hline Age & 1.06 & $1.01-1.11$ & 0.030 & - & - & - \\
\hline \multicolumn{7}{|c|}{ In the HFpEF population } \\
\hline BMl & 1.20 & $1.07-1.35$ & 0.003 & - & - & - \\
\hline Systemic hypertension & 2.99 & $1.14-7.87$ & 0.030 & 2.84 & $1.07-7.57$ & 0.040 \\
\hline
\end{tabular}

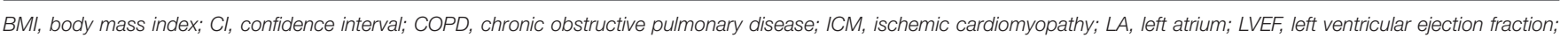
NYHA, New York Heart Association; OR, odds ratio.

$p<0.05)$. No significant difference was observed in patients with HFmrEF (all $p>0.05$ ).

Analysis of clinical correlates of OA was only limited at nighttime given their low prevalence at daytime (Supplementary Table 3). When compared to NB, patients with nighttime $\mathrm{OA}$ had higher prevalence of comorbidities (obesity and hypertension in HFrEF, hypertension in HFpEF; all $p<0.05$ ) and echocardiographic abnormalities (higher LA volume in HFrEF and higher sPAP in HFmrEF; all $p<0.05$ ), despite a similar degree of adrenergic activation (all $p>0.05)$.

\section{DISCUSSION}

To our knowledge, this is the first study to address the prevalence, clinical predictors and clinical correlates of both $\mathrm{OA}$ and CA throughout the entire 24-h period and across the whole spectrum of HF. Patients with HFrEF had higher prevalence of CA than patients with HFmrEF and HFpEF, while $\mathrm{OA}$ were more common in patients with HFpEF. In HFrEF patients, specific predictors of $\mathrm{CA}$ and $\mathrm{OA}$ were identified (male gender and BMI for nighttime CA and OA; age, NYHA class and diastolic dysfunction for daytime CA), while no predictors were found in the HFmrEF and HFpEF populations (besides sPAP as predictor for CA in patients with HFmrEF). When compared to NB, patients with CA had higher sympathetic activation (as demonstrated by increased plasmatic norepinephrine levels), greater NT-proBNP levels and increased indexes of hemodynamic overload in each HF subgroups (i.e., higher LA volume and worse diastolic function in HFrEF, increased LA volume in HFmrEF and worse diastolic function in $\mathrm{HFpEF}$ ). On the other hand, OA patients were more comorbid (in patients with HFrEF and HFpEF), had a worse hemodynamic profile (increased LA volume and sPAP in HFrEF and HFmrEF, respectively), but similar degree of neuro-hormonal activation as compared to patients with NB.

In the HFrEF population, the prevalence and prognostic significance of CA has been largely established both during the day and at night $(2,8,25-27)$. In particular, patients presenting with CA throughout the 24-h (including the daytime) were shown to have an increased risk of mortality when compared with patients presenting with CA only at nighttime (2). In our study, approximately two thirds of patients presented with CA during the night, while CA were observed in about half of the population at daytime (using a AHI threshold of 5 events/h). Higher prevalence of nighttime CA over daytime CA, as observed in the current study, can be related to the loss of cortical influences and predominance of feed-back control on respiration at night, as well as to rostral fluid shift occurring in the recumbent position typical of patients with HF (28-30).

Daytime CA has been previously described in smaller studies performed in systolic HF patients with short term recordings (10 or $20 \mathrm{~min})(5,6)$, finding a prevalence ranging from 38 to $59 \%$. Those preliminary findings were later confirmed with $24-\mathrm{h}$ portable systems first in a smaller population by Brack et al. 
TABLE 6 | Clinical comparison between nighttime only and combined daytime and nighttime moderate-severe CA across the whole HF spectrum.

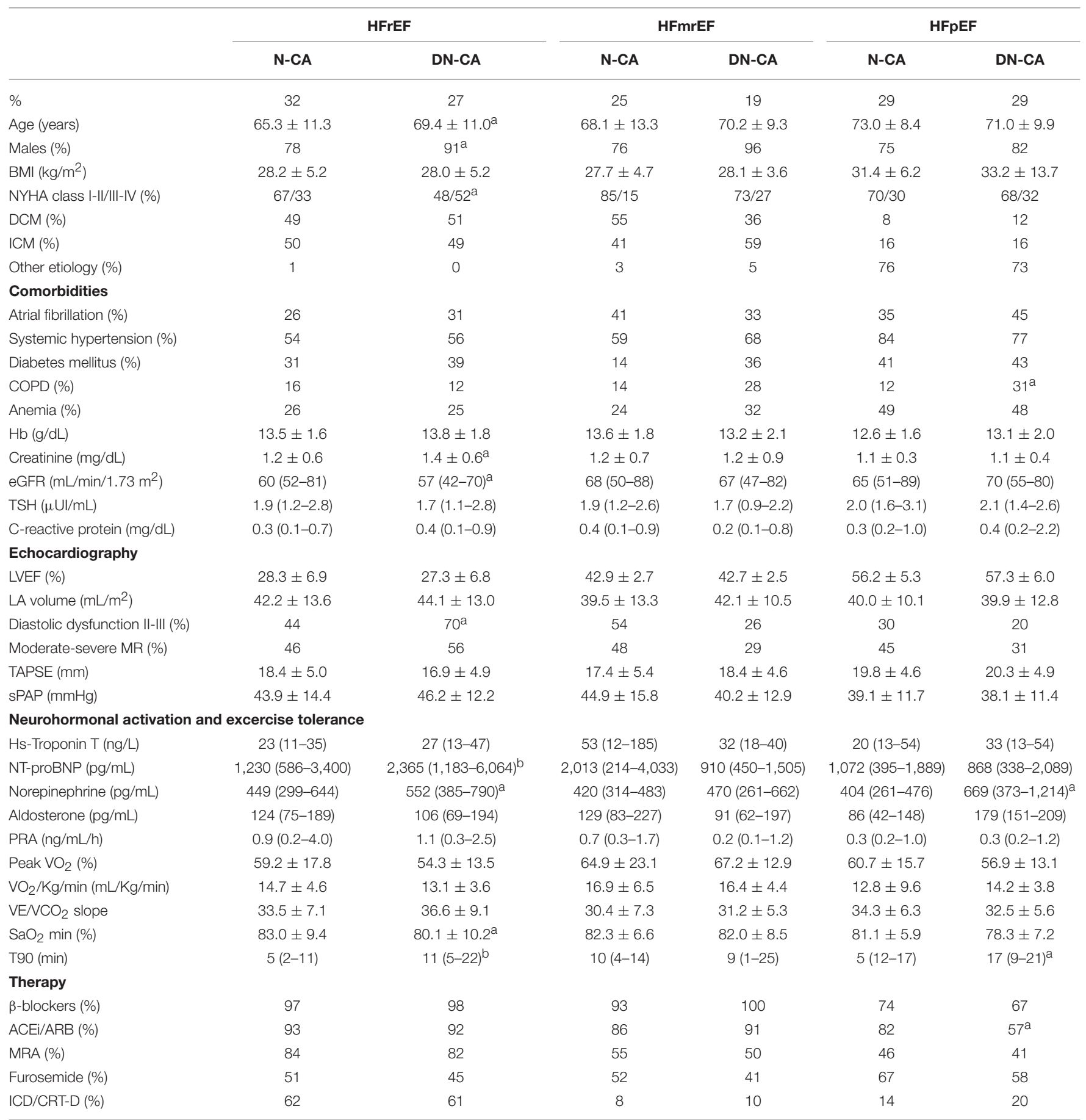

${ }^{a} p<0.05 N-C A$ vs. DN-CA.

${ }^{b} p<0.001 \mathrm{~N}-C A$ vs. DN-CA.

${ }^{*}$ Apneas were defined as moderate-severe (AHI $\geq 15$ events/h). Patients were classified according to the presence of only nighttime apneas (N-CA) or the presence of both nighttime and daytime apneas (DN-CA).

ACEi, angiotensin-converting enzyme inhibitors; ARB, angiotensin II receptor blockers; BMI, body mass index; COPD, chronic obstructive pulmonary disease; CRT-D, cardiac resynchronization therapy with defibrillator; DCM, dilated cardiomyopathy; eGFR, estimated glomerular filtration rate (with the Modification of Diet in Renal Disease-MDRD formula); Hb, hemoglobin; Hs, high sensitive; ICD, implantable cardioverter defibrillator; ICM, ischemic cardiomyopathy; LA, left atrium; LVEF, left ventricular ejection fraction; LVMI, left ventricular mass index; MR, mitral regurgitation; MRA, mineralocorticoid receptor antagonists; NT-proBNP, N-terminal fraction of pro-B-type natriuretic peptide; NYHA, New York Heart Association; PRA, plasmatic renin activity; $\mathrm{SaO}_{2}$ :oxygen saturation; SPAP, systolic pulmonary artery pressure; T90, time spent with an oxygen saturation below 90\%; TAPSE, tricuspid annular plane systolic excursion; TSH, thyroid stimulating hormone; $\mathrm{VENCO}_{2}$, ventilation to carbon dioxide production ratio; $\mathrm{VO}_{2}$, oxygen consumption. 
(10), and then in a larger population by our group (2). Those studies also highlighted that patients with both nighttime and daytime CA have the highest risk of experiencing life-threatening events $(2,10)$. In our study, HFrEF patients with CA (moderatesevere, AHI $>15$ events/h) throughout the 24-h (at daytime and nighttime) showed increased neuro-hormonal activation and worse diastolic and $\mathrm{SaO}_{2}$ profiles (higher T90, an independent prognostic marker) (7) than patients with CA occurring only at nighttime, thus providing pathophysiological insight to previous prognostic observations $(2,7)$. Higher T90 and norepinephrine plasma levels were found also in HFpEF patients with CA over the 24-h, suggesting that this subset of patients might be at increased risk of mortality, as already documented in HFrEF.

Of note, a greater diastolic impairment was found to be predictive of daytime CA in our study at least in HFrEF patients. Increased ventricular stiffening and impaired relaxation, together with increased LV and LA pressure, might in fact be responsible of chemoreflex activation via pulmonary J-receptor or C-fibers stimulation (31-33), thus promoting ventilatory instability, as already demonstrated in a canine model of LA pressure augmentation via balloon inflation (34).

In the HFrEF population, the prevalence of $\mathrm{OA}$ found in this study was similar to that found by Grimm and coworkers (35) while lower than other previous reports (36-38). Differences in patients' characteristics, study design (retrospective vs. prospective), apnea definition (AHI threshold) and detection (attended standard polysomnography vs. portable long-term ambulatory systems), and HF severity (i.e., greater diastolic dysfunction and/or MR) might help explain those discrepancies.

In HFpEF, on the other hand, we found that approximately one third of the patients presented with nighttime CA, while nighttime OA was generally more prevalent, being observed in one patient out of two. The higher prevalence of OA in patients with HFpEF may simply express the high prevalence of OA in the general population ( $>20 \%$ in subjects with over the age of 60) (39): those subjects may become patients with HFpEF over time (higher age compared to HFmrEF and HFrEF), due to OA related adrenergic overactivation, phasic hypoxia-reoxigenation driving to oxidative stress and inflammation and intra-pleural pressure swings (40). On the other hand, CA have been described at night also in patients with $\operatorname{HFpEF}(18,25)$ and tend to emerge whenever the diastolic profile deteriorates (18). This also justifies the higher prevalence of nocturnal CA in patients hospitalized for HF (30\%) (18-25), compared to the outpatient scenario (18\%) (19), due to the increased clinical and haemodynamic stability in the latter case.

Daytime CA, on the other hand, have never been described in HFpEF. Interestingly, we found that $42 \%$ of HFpEF patients present with daytime CA, a prevalence slightly lower than the one observed in patients with HFrEF.

On the contrary, during the daytime OA prevalence is much lower (18\%). This may be associated with a minor contribution of anatomical factors (such as obesity and increased fat deposition around the upper airway) as compared to haemodynamic or neural factors (worse diastolic profile with J-receptor stimulation, high loop-gain OA transforming to CA during the day) in awake and standing conditions (30-34, 41). Alternatively, reduced lung volume and increased prevalence of pulmonary and metabolic comorbidities in patients with OA can also impact on the plant (or the lung) gain during the day, thus promoting a shift from OA to CA (42).

Finally, we described CA in patients with HFmrEF both at nighttime (48\%) and at daytime (43\%). The apnea prevalence in HFmrEF has never been previously reported, and its prognostic significance still needs to be clarified, as it has only been addressed in mixed population of HFrEF and HFmrEF patients (patients with systolic HF) $(8,26,38)$.

Nonetheless, we found that HFmrEF patients with CA have greater LA volumes, which can again suggest a potential role of backward failure with increased filling pressure, rather than decreased cardiac output, as the main mechanism leading to ventilatory instability in this setting $(34,43)$.

Presence of daytime CA in the HFmrEF population was associated with increased SPAP in this study. Whether increased sPAP is a cause or a consequence of CA is still unclear. However, it seems that increased sPAP could be ascribed to vasoconstriction of pulmonary vessels due to chemoreflex overactivation even in presence of only slightly depressed LVEF (44, 45). Similarly, intermittent hypoxia may directly cause adverse vascular remodeling on the long term, thus increasing average pulmonary pressures beyond dynamic variations due to alternating phases of CA and hyperventilation (44-46).

Nonetheless, both daytime and nighttime CA were associated with higher NT-proBNP and plasma norepinephrine values despite LVEF in this study, while plasmatic levels of norepinephrine and NT-proBNP activation did not differ between $\mathrm{OA}$ and $\mathrm{NB}$, partially explaining the lower prognostic impact of OA as compared to CA in HF.

Of note, this is the first study describing CA as a 24-h phenomenon in spite of LVEF classification in HF. Therefore, screening for CA with tools assessing both daytime and nighttime apneas would be advisable. Similarly, therapeutic approaches that also challenge daytime CA seem desirable in patients with HFrEF, considering the prognostic significance of daytime apneas, (2) and they might be advisable also in patients with HFpEF, considering the clinical correlates associated with daytime CA observed in the current study.

\section{STUDY LIMITATIONS}

Hypopneas were scored according to the main trend of central or obstructive events, as previously reported (24), which could lead to underestimation of misclassification of the events. However, invasive maneuvers such as transoesophageal pressure transducer or diaphragmatic electromyography are both unfeasible over the 24 -h and unpractical in a large series of patients.

For similar reasons, we chose to use an unattended ambulatory system to screen for CA/OA, rather than performing a standard polysomnography. Despite losing information about cortical activity (essential to define the sleep/awake periods), the ambulatory system has the advantage of allowing a 24-h recording, with a diagnostic accuracy comparable to standard polysomnography $(21,22)$. This information, especially if supported by prognostic data (still to be acquired), will 
presumably change both diagnostic screening algorithms and the therapeutical approaches, including the daytime period at least when dealing with CA in HF.

Finally, a comprehensive evaluation of neuroreflexes is also lacking, especially when addressing pathophysiological mechanisms and prognosis of the respiratory disease. Further studies are needed to clarify their relative contribution to the pathophysiology and prognostic significance of CA/OA in the different HF phenotypes, also with the aim of developing tailored and rational treatments.

\section{CONCLUSIONS}

This is the first study to describe the prevalence of both OA and CA during the whole $24-\mathrm{h}$ period and, more importantly, across the whole HF spectrum. Both CA and OA are highly prevalent phenomena regardless of LVEF and can be detected both at nighttime and daytime, with CA prevalence increasing and OA prevalence decreasing as LV function worsens.

We also analyzed the clinical predictors and correlates of $\mathrm{CA}$ and $\mathrm{OA}$ in $\mathrm{HF}$ and found that $\mathrm{CA}$ are associated with worse hemodynamic profile (diastolic dysfunction, LA volume and sPAP), as well as worse symptoms, especially in HFrEF, while $\mathrm{OA}$ are associated with higher degree of comorbidities, especially obesity and hypertension. Those data suggest specific pathophysiological mechanisms underlying $\mathrm{CA}$ and $\mathrm{OA}$ in different HF categories and potentially different therapeutic targets. Interestingly, both nighttime and daytime CA were associated with worse neuro-hormonal activation, as opposed to OA, partially explaining the worse prognosis observed in patients with CA and identifying a high-risk subset in which

\section{REFERENCES}

1. Ponikowski P, Voors AA, Anker SD, Bueno H, Cleland JGF, Coats AJS, et al. 2016 ESC Guidelines for the diagnosis and treatment of acute and chronic heart failure: the Task Force for the diagnosis and treatment of acute and chronic heart failure of the European Society of Cardiology (ESC). Developed with the special contribution of the Heart Failure Association (HFA) of the ESC. Eur J Heart Fail. (2016) 18:891-975. doi: 10.1093/eurheartj/ehw128

2. Emdin M, Mirizzi G, Giannoni A, Poletti R, Iudice G, Bramanti F, et al. Prognostic significance of central apneas throughout a 24-Hour period in patients with heart failure. J Am Coll Cardiol. (2017) 70:1351-64. doi: 10.1016/j.jacc.2017.07.740

3. Yumino D, Wang H, Floras JS, Newton GE, Mak S, Ruttanaumpawan P, et al. Prevalence and physiological predictors of sleep apnea in patients with heart failure and systolic dysfunction. J Card Fail. (2009) 15:279-85. doi: 10.1016/j.cardfail.2008.11.015

4. Lanfranchi PA, Braghiroli A, Bosimini E, Mazzuero G, Colombo R, Donner $\mathrm{CF}$, et al. Prognostic value of nocturnal Cheyne-Stokes respiration in chronic heart failure. Circulation. (1999) 99:1435-40. doi: 10.1161/01.CIR.99.11.1435

5. Poletti R, Passino C, Giannoni A, Zyw L, Prontera C, Bramanti F, et al. Risk factors and prognostic value of daytime Cheyne-Stokes respiration in chronic heart failure patients. Int J Cardiol. (2009) 137:47-53. doi: 10.1016/j.ijcard.2008.06.028

6. La Rovere MT, Pinna GD, Maestri R, Robbi E, Mortara A, Fanfulla F, et al. Clinical relevance of short-term day-time breathing disorders in chronic heart failure patients. Eur J Heart Fail. (2007) 9:949-54. doi: 10.1016/j.ejheart.2007.06.009 treatment seems to be advisable to also obtain favorable effects on HF progression.

\section{DATA AVAILABILITY}

The datasets generated for this study are available on motivated request to the corresponding author.

\section{ETHICS STATEMENT}

The studies involving human participants were reviewed and approved by Comitato Etico Area Vasta Nord Ovest (CEAVNO) Toscana, Italy. The patients/participants provided their written informed consent to participate in this study.

\section{AUTHOR CONTRIBUTIONS}

$\mathrm{CB}, \mathrm{FG}, \mathrm{PS}, \mathrm{GV}, \mathrm{NG}$, and GM contributed to data collecting and to the formation of a comprehensive database for analysis. $\mathrm{FB}$ and GI contributed to analysis of 24-h recordings as expert sleep technicians. $\mathrm{CB}, \mathrm{AG}, \mathrm{CP}$, and $\mathrm{ME}$ contributed to the expert revision of $24-\mathrm{h}$ recordings. $\mathrm{CB}, \mathrm{FG}, \mathrm{PS}$, and AG contributed to data analysis, to manuscript writing and production of figures and tables. AG, CP, and ME contributed to manuscript revising.

\section{SUPPLEMENTARY MATERIAL}

The Supplementary Material for this article can be found online at: https://www.frontiersin.org/articles/10.3389/fcvm. 2019.00125/full\#supplementary-material

7. Oldenburg O, Wellmann B, Buchholz A, Bitter T, Fox H, Thiem U, et al. Nocturnal hypoxaemia is associated with increased mortality in stable heart failure patients. Eur Heart J. (2016) 37:1695-703. doi: 10.1093/eurheartj/ehv624

8. Grimm W, Sosnovskaya A, Timmesfeld N, Hildebrandt O, Koehler U. Prognostic impact of central sleep apnea in patients with heart failure. J Card Fail. (2015) 21:126-33. doi: 10.1016/j.cardfail.2014. 10.017

9. Pinna GD, Maestri R, Mortara A, Johnson P, Andrews D, Ponikowski $\mathrm{P}$, et al. Pathophysiological and clinical relevance of simplified monitoring of nocturnal breathing disorders in heart failure patients. Eur J Heart Fail. (2009) 11:264-72. doi: 10.1093/eurjhf/ hfp006

10. Brack T, Thuer I, Clarenbach CF, Senn O, Noll G, Russi EW, et al. Daytime Cheyne-Stokes respiration in ambulatory patients with severe congestive heart failure is associated with increased mortality. Chest. (2007) 132:1463-71. doi: 10.1378/chest.07-0121

11. Arzt M, Woehrle H, Oldenburg O, Graml A, Suling A, Erdmann E, et al. SchlaHF Investigators. Prevalence and predictors of sleepdisordered breathing in patients with stable chronic heart failure: the SchlaHF Registry. JACC Heart Fail. (2016) 4:116-25. doi: 10.1016/j.jchf.2015. 09.014

12. Herrscher TE1, Akre H, Overland B, Sandvik L, Westheim AS. Clinical predictors of sleep apnoea in heart failure outpatients. Int J Clin Pract. (2014) 68:725-30. doi: 10.1111/ijcp.12396

13. Lanfranchi PA, Somers VK, Braghiroli A, Corra U, Eleuteri E, Giannuzzi P. Central sleep apnea in left ventricular dysfunction: prevalence 
and implications for arrhythmic risk. Circulation. (2003) 11:727-32. doi: 10.1161/01.CIR.0000049641.11675.EE

14. Mehra R, Redline S. Arrhythmia Risk Associated with Sleep Disordered Breathing in Chronic Heart Failure. Curr Heart Fail Rep. (2014) 11:88-97. doi: 10.1007/s11897-013-0171-7

15. Oldenburg O, Lamp B, Faber L, Teschler H, Horstkotte D, Töpfer V. Sleep-disordered breathing in patients with symptomatic heart failure. A contemporary study of prevalence in and characteristics of 700 patients. Eur $J$ Heart Fail. (2007) 9:251-7. doi: 10.1016/j.ejheart.2006.08.003

16. Lipatov K, Hayek A, Ghamande S, Boethel C, Chen W, Jones S. Predictors of obstructive sleep apnea on a home sleep apnea test after a negative attended polysomnography. J Clin Sleep Med. (2018) 14:1889-94. doi: $10.5664 /$ jcsm. 7486

17. Bitter T, Westerheide N, Prinz C, Hossain MS, Vogt J, Langer C, et al. CheyneStokes respiration and obstructive sleep apnoea are independent risk factors for malignant ventricular arrhythmias requiring appropriate cardioverterdefibrillator therapies in patients with congestive heart failure. Eur Heart J. (2011) 32:61-74. doi: 10.1093/eurheartj/ehq327

18. Bitter T, Faber L, Hering D, Langer C, Horstkotte D, Oldenburg O. Sleep-disordered breathing in heart failure with normal left ventricular ejection fraction. Eur J Heart Fail. (2009) 11:602-8. doi: 10.1093/eurjhf/ hfp057

19. Herrscher TE, Akre H, Øverland B, Sandvik L, Arne S. Westheim A. High prevalence of sleep apnea in heart failure outpatients: even in patients with preserved systolic function. J Cardiac Fail. (2011) 5:420-5. doi: 10.1016/j.cardfail.2011.01.013

20. Giannoni A, Emdin M, Bramanti F, Iudice G, Francis DP, Barsotti A, et al. Combined increased chemosensitivity to hypoxia and hypercapnia as a prognosticator in heart failure. J Am Coll Cardiol. (2009) 53:1975-80. doi: 10.1016/j.jacc.2009.02.030

21. Kapur VK, Auckley DH, Chowdhuri S, Kuhlmann DC, Mehra R, Ramar $\mathrm{K}$, et al. Clinical practice guideline for diagnostic testing for adult obstructive sleep apnea: an American Academy of Sleep Medicine clinical practice guideline. J Clin Sleep Med. (2017) 13:479-504. doi: 10.5664/jcsm. 6506

22. Somers VK, White DP, Amin R, Abraham WT, Costa F, Culebras A, et al. Sleep apnea and cardiovascular disease: an American Heart Association/American College of Cardiology Foundation Scientific Statement from the American Heart Association Council for High Blood Pressure Research Professional Education Committee, Council on Clinical Cardiology, Stroke Council, and Council on Cardiovascular Nursing. J Am Coll Cardiol. (2008) 52:686-717. doi: 10.1161/CIRCULATIONAHA.107.189420

23. Randerath W, Verbraecken J, Andreas S, Arzt M, Bloch KE, Brack T, et al. Definition, discrimination, diagnosis and treatment of central breathing disturbances during sleep. Eur Respir J. (2017) 49:1600959. doi: 10.1183/13993003.00959-2016

24. Ryan CM, Floras JS, Logan AG, Kimoff RJ, Series F, Morrison D, et al. CANPAP Investigators. Shift in sleep apnoea type in heart failure patients in the CANPAP trial. Eur Respir J. (2010) 35:592-7. doi: 10.1183/09031936.00070509

25. Sekizuka H, Osada N, Miyake F. Sleep disordered breathing in heart failure patients with reduced versus preserved ejection fraction. Heart Lung Circul. (2013) 22:104-9. doi: 10.1016/j.hlc.2012.08.006

26. Jilek C, Krenn M, Sebah D, Obermeier R, Braune A, Kehl V, et al. Prognostic impact of sleep disordered breathing and its treatment in heart failure: an observational study. Eur J Heart Fail. (2011) 13:68-75. doi: 10.1093/eurjhf/hfq183

27. Javaheri S, Shukla R, Zeigler H, Wexler L. Central sleep apnea, right ventricular dysfunction, and low diastolic blood pressure are predictors of mortality in systolic heart failure. J Am Coll Cardiol. (2007) 49:2028-34. doi: 10.1016/j.jacc.2007.01.084

28. Yumino D, Redolfi S, Ruttanaumpawan P, Su MC, Smith S, Newton GE, et al. Nocturnal rostral fluid shift: a unifying concept for the pathogenesis of obstructive and central sleep apnea in men with heart failure. Circulation. (2010) 121:1598-605. doi: 10.1161/CIRCULATIONAHA.109. 902452

29. Joho S, Oda Y, Hirai T, Inoue H. Impact of sleeping position on central sleep apnea/Cheyne-Stokes respiration in patients with heart failure. Sleep Med. (2010) 11:143-8. doi: 10.1016/j.sleep.2009.05.014
30. Sahlin C, Svanborg E, Stenlund H and Franklin KA. Cheyne-Stokes respiration and supine dependency. Eur Respir J. (2005) 25:829-33. doi: 10.1183/09031936.05.00107904

31. Paintal AS, Ravi K. The relative location of lowand higher-threshold pulmonary stretch receptor. J Physiol. (1985) 307:50.

32. Feldman JL, McCrimmon DR, Morrison SF. Neural Control of respiratory and cardiovascular functions. In: Squire LR, Berg D, Bloom FE, Ghosh S, du Lac S, Spitzer NC, editors. Fundamental Neuroscience, 4th edition. Cambridge, MA: Academic Press (2013). p. 749-66.

33. Anand A. Influence of bronchial C fibre receptors on respiration in cats: possible role in humans. Resp Physiol. (2000) 123:1-12. doi: 10.1016/S0034-5687(00)00137-7

34. Chenuel BJ, Smith CA, Skatrud JB, Henderson KS, Dempsey JA. Increased propensity for apnea in response to acute elevations in left atrial pressure during sleep in the dog. J Appl Physiol. (2006) 101:76-83. doi: 10.1152/japplphysiol.01617.2005

35. Grimm W, Apelt S, Timmesfeld N, Koehler U. Sleep-disordered breathing in patients with implantable cardioverter-defibrillator. Europace. (2013) 15:51522. doi: 10.1093/europace/eus356

36. MacDonald M, Fang J, Pittman SD, White DP, Malhotra A. The current prevalence of sleep disordered breathing in congestive heart failure patients treated with beta-blockers. J Clin Sleep Med. (2008) 4:38-42.

37. Oldenburg O, Lamp B, Freudenberg G, Horstkotte D. Screening for sleepdisordered breathing is recommended in patients with chronic heart failure. Eur Respir J. (2007) 30:1023-4. doi: 10.1183/09031936.00073407

38. Damy T, Margarit L, Noroc A, Bodez D, Guendouz S, Boyer L, et al. Prognostic impact of sleep-disordered breathing and its treatment with nocturnal ventilation for chronic heart failure. Eur J Heart Fail. (2012) 14:1009-19. doi: 10.1093/eurjhf/hfs085

39. Young T, Shahar E, Nieto FJ, Redline S, Newman AB, Gottlieb DJ, et al. Predictors of sleep-disordered breathing in community-dwelling adults: the Sleep Heart Health Study. Arch Intern Med. (2002) 162:893-900. doi: 10.1001/archinte.162.8.893

40. Dewan NA, Nieto FJ, Somers VK. Intermittent hypoxemia and OSA. Implications for Comorbidities. Chest. (2015) 147:266-74. doi: 10.1378/chest.14-0500

41. Burgess KR. New insights from the measurement of loop gain in obstructive sleep apnoea. J Physiol. (2012) 590:1781-2. doi: 10.1113/jphysiol.2012.228643

42. Giannoni A, Gentile F, Navari A, Borrelli C, Mirizzi G, Catapano G et al. Contribution of the lung to the genesis of Cheyne-Stokes respiration in heart failure: plant gain beyond chemoreflex gain and circulation time. J Am Heart Ass. (2019) 8:e012419. doi: 10.1161/JAHA.119.012419

43. Cameli M, Mandoli G, Mondillo S. Left atrium: the last bulwark before overt heart failure. Heart Fail Rev. (2017) 22:123-31. doi: 10.1007/s10741-016-9589-9

44. Giannoni A, Raglianti V, Mirizzi G, Taddei C, Del Franco A, Iudice G et al. Influence of central apneas and chemoreflex activation on pulmonary artery pressure in chronic heart failure. Int J Cardiol. (2016) 202:200-6. doi: 10.1016/j.ijcard.2015.09.007

45. Giannoni A, Raglianti V, Taddei C, Borrelli C, Chubuchny V, Vergaro $G$ et al. Cheyne-Stokes respiration related oscillations in cardiopulmonary hemodynamics in patients with heart failure. Int $J$ Cardiol. (2019) S0167-5273:30986-6. doi: 10.1016/j.ijcard.2019.03.033

46. Javaheri S, Javaheri S, Javaheri A. Sleep apnea, heart failure, and pulmonary hypertension. Curr Heart Fail Rep. (2013) 10:315-20. doi: 10.1007/s11897-013-0167-3

Conflict of Interest Statement: The authors declare that the research was conducted in the absence of any commercial or financial relationships that could be construed as a potential conflict of interest.

Copyright (c) 2019 Borrelli, Gentile, Sciarrone, Mirizzi, Vergaro, Ghionzoli, Bramanti, Iudice, Passino, Emdin and Giannoni. This is an open-access article distributed under the terms of the Creative Commons Attribution License (CC BY). The use, distribution or reproduction in other forums is permitted, provided the original author(s) and the copyright owner(s) are credited and that the original publication in this journal is cited, in accordance with accepted academic practice. No use, distribution or reproduction is permitted which does not comply with these terms. 